\title{
Orthogonality relations for triple modes at dielectric boundary surfaces
}

\author{
Hanno Hammer ${ }^{\text {f }}$ \\ Department of Chemical Physics, \\ The Weizmann Institute of Science, \\ 76100 Rehovot, Israel
}

(Dated: 30. Nov. 2001)

\begin{abstract}
We work out the orthogonality relations for the set of Carniglia-Mandel triple modes which provide a set of normal modes for the source-free electromagnetic field in a background consisting of a passive dielectric half-space and the vacuum, respectively. Due to the inherent computational complexity of the problem, an efficient strategy to accomplish this task is desirable, which is presented in the paper. Furthermore, we provide all main steps for the various proofs pertaining to different combinations of triple modes in the orthogonality integral.
\end{abstract}

\section{INTRODUCTION}

The electromagnetic field propagating between boundary surfaces of conducting or dielectric media differs from the free electromagnetic field in vacuum in that the boundary conditions imposed by the material media on the field alter the structure of the normal modes of the field as well as the state density of electromagnetic field modes. The alteration of these quantities becomes manifest when transition rates and energy level shifts of atomic matter exposed to the field environment are studied, as these quantities directly enter the expressions of the relevant transition matrix elements.

The complex of problems relating to these ideas is now generally called Cavity Quantum Electrodynamics (CQED). One of the principal ideas underlying CQED appeared first in the abstract of paper [1]. CQED as a subject has been initiated in [2] with the study of atoms coupled to a single quantized mode of the radiation field in a cavity, followed by articles [3] and [4] working on modifications of spontaneous decay rates and radiative level shifts of optical transitions in a cavity. Resonators, waveguides, dielectrics, and conducting interfaces modifying atomic radiative properties such as decay times, shifts in energy levels, and spectral line shapes have been reviewed in [5]. A review of the subject of CQED in the presence of metallic boundaries including exhaustive references to original papers was given in [6].

The mode structure as well as the problem of atomic excitation and photoemission by evanescent waves at a dielectric half-space have been derived at first in [7, 8]. There it was found that an appropriate set of normal modes was given by so-called triple modes, which are composed of three plane-wave components (incoming, reflected, transmitted). The transmitted component of a triple mode can be a travelling wave or an evanescent wave, with exponentially decreasing amplitude behind the boundary plane. A variant of these modes has been used in [9], where the radiation properties of a quantum harmonic oscillator near a planar dielectric half-space were examined. In [10], special linear combinations of time-reversed and spatially rotated Carniglia-Mandel triple modes have been employed to determine spontaneous emission rates and final-state mode densities. A further generalization has been presented in [11, 12], where spontaneous emission in the presence of a dielectric slab bounded by two dielectric half-spaces was studied. General quantum optics with the main focus on quantum fluctuations in dielectric media was discussed in [13].

One of the central problems in these developments is the determination of an appropriate set of normal modes of the free electromagnetic field subject to the given boundary conditions. These normal modes obey the sourcefree Maxwell equations in a dielectric and satisfy the boundary conditions describing either conducting or dielectric surfaces. Having solved for a set of modes it is crucial to check their orthogonality, as this property determines whether the free field Hamiltonian can be diagonalized in these modes.

The question of orthogonality of normal modes is simple only in the case of no boundaries, i.e. the field in vacuum. As soon as boundaries are imposed, checking for orthogonality can be a tedious procedure. For this reason, actual computations proving the orthogonality of modes currently used in the literature are not widely available. For example, even in the seemingly simple problem of a dielectric half-space the question of orthogonality of the triple modes becomes highly non-trivial. In particular, orthogonality of a pair of triple modes containing a traveling and an evanescent component in the same half-space, respectively, is quite counter-intuitive. A brief proof of orthogonality in the case of a dielectric half-space has first been given in the appendix of [7]. We feel, however, that there is a demand for a more detailed examination of this problem.

*Electronic address: H.Hammer@umist.ac.uk 
Our work presented here is intended to fill this gap. We expand on the results presented in the appendix of [7], and give a proof of orthogonality for all different cases of triple modes as they appear in the dielectric half-space problem. Each proof attempts to highlight the most important intermediate steps as well as the mathematical tools necessary to derive them. We do not enter the issue of quantization of these modes, but will end by showing that the free field Hamiltonian can be diagonalized in these modes and hence can be written as a sum of independent harmonic oscillators pertaining to these triple modes, within a classical context. The completeness of the set of triple modes in the space of transverse vector-valued functions has been proven in [14]. This, together with our result, shows that the triple modes are actually 'good' modes for a canonical quantization of the electromagnetic field in a dielectric half-space.

In this approach, the triple modes appear as traveling normal modes of the free electromagnetic field. The term 'free' deserves some explanation here: Strictly speaking, propagation of an electromagnetic plane wave through a material medium is the result of the interaction of the fundamental fields $\mathbf{E}$ and $\mathbf{B}$ with a macroscopic number of microscopic sources constituting the bulk matter. In principle, these sources must be incorporated into the dynamics of the total system by suitable interaction terms. However, if the frequency band width of the plane waves under consideration is far off the resonance frequencies of the bulk matter, any light-matter interaction will be only transient in nature, and will certainly not affect the macroscopic state of the bulk matter. In this case, we can treat the bulk matter as a passive medium, whose macroscopic properties with respect to the interaction with radiation can be summarized by introducing a (space- and time-dependent) refractive index. In the following we will use this approach.

\section{TRIPLE MODES}

The Carniglia-Mandel modes are assumed to propagate in a background which consists of a non-magnetic material medium with refractive index $n=\sqrt{\frac{\epsilon \mu_{0}}{\epsilon_{0} \mu_{0}}}$ in the left half-space $z<0$, and the vacuum with refractive index $n=1$ in the right half-space $z>0$, where $\epsilon, \epsilon_{0}$ are the permittivities of the medium and the vacuum, respectively, and $\mu_{0}$ is the uniform magnetic permeability which takes the vacuum value everywhere, since the medium is non-magnetic. Furthermore, $\epsilon$ is assumed to be time-independent.

Before we state the form of the triple modes, we introduce our notation conventions. Let $\mathbf{e}_{3}$ be the unit normal vector onto the boundary plane (i.e. the plane extends in the $x y$-directions), then any vector $\mathbf{V}$ on the boundary can be decomposed into components parallel and perpendicular to the boundary plane $\mathbf{V}=\mathbf{V}_{\|}+\mathbf{V}_{\perp}$. In particular, the wave vector $\mathbf{k}$ of a homogeneous plane wave incident on the boundary plane can be decomposed as $\mathbf{k}=K \mathbf{e}_{3}+\mathbf{k}_{\|}$, where $\mathbf{k} \bullet \mathbf{e}_{3} \equiv K$. In the following, this notation will be used extensively.

The triple modes are obtained by making a separation ansatz for the electric/magnetic fields, factoring out the time dependence in the form of $e^{-i \omega t}$. A typical electric field will then take the form $\mathbf{E}(\mathbf{k}, \mathbf{x}) e^{-i \omega t}$, where the spatial part $\mathbf{E}(\mathbf{k}, \mathbf{x})$ depends on the wave vector $\mathbf{k}$ and satisfies an appropriate Helmholtz equation.

The triple modes can be distinguished by their polarization with respect to the incident plane, which is spanned by the incoming wave vector $\mathbf{k}_{i}$ and the normal vector $\mathbf{e}_{3}$. If the incoming electric field $\mathbf{E}_{i}$ is perpendicular to the incident plane, the mode is called transverse-electric (TE); this will be indicated by polarization index 1 . If the incoming electric field lies in the plane of incidence, the mode is called transverse-magnetic (TM), indicated by polarization index 2. Each triple mode is composed of an incoming, a reflected and a transmitted plane wave component. We shall label all quantities pertaining to these components by subscripts $i, r, t$, respectively. Furthermore, fields will carry a subscript $L$ or $R$ depending on whether the corresponding incoming wave comes from the left, $K_{i}>0$, or from the right, $K_{i} \leq 0$.

To simplify the subsequent computations we first choose normalization conventions different from those in [7]: we assign unit amplitude to incoming electric plane waves in the TE case, and unit amplitude to incoming magnetic plane waves in the TM case. Only at the end of our work shall we discuss a different normalization appropriate for the diagonalization of the field Hamiltonian.

The TE modes incoming from the left are labelled by $L$, the incoming wave vector $\mathbf{k}_{i}$, and the polarization index 1 , and are given by their electric field components

$$
\mathbf{E}_{L}\left(\mathbf{k}_{i}, 1, \mathbf{x}\right)=\left\{\begin{array}{cl}
\mathbf{e}\left[e^{i \mathbf{k}_{i} \bullet \mathbf{x}}+a_{r} e^{i \mathbf{k}_{r} \bullet \mathbf{x}}\right] & , z<0 \\
\mathbf{e} a_{t} e^{i \mathbf{k}_{t} \bullet \mathbf{x}} & , z \geq 0
\end{array},\right.
$$

where the real unit polarization vector $\mathbf{e}$ is chosen in the direction of $\mathbf{k}_{i} \times \mathbf{e}_{3}$, and the incoming wave is assumed to have unit amplitude. $\mathbf{k}_{r}$ is the wave vector of the reflected wave, with $K_{r}=-K_{i}$, and $\mathbf{k}_{t}$ is the wave vector of the transmitted wave; its component normal to the boundary $K_{t}$ can be real or imaginary, depending on the angle of the incoming wave; in the latter case, the triple mode is called evanescent. The three components of the mode must (together with their time dependence $e^{-i \omega t}$ ) satisfy the Maxwell equations on the boundary 15 as well as a phase 
matching condition. This determines the reflexion / transmission coefficients $a_{r}, a_{t}$ to be

$$
a_{r}=\frac{K_{i}-K_{t}}{K_{i}+K_{t}} \quad, \quad a_{t}=\frac{2 K_{t}}{K_{i}+K_{t}} .
$$

For modes incoming from the left, frequency $\omega$ and wave vectors $\mathbf{k}$ are related by

$$
\mathbf{k}_{\|}^{2}+K_{i}^{2}=\mathbf{k}_{\|}^{2}+K_{r}^{2}=\mu_{0} \epsilon \omega^{2} \quad, \quad \mathbf{k}_{\|}^{2}+K_{t}^{2}=\mu_{0} \epsilon_{0} \omega^{2} .
$$

We note that $K_{i}$ and $K_{r}$ are always real, but the fact that $\epsilon>\epsilon_{0}$ allows for $K_{t}$ to be real or imaginary; in the latter case, the transmitted wave is evanescent. The associated TE magnetic field is uniquely determined by Maxwell's equations, and is given by

$$
\mathbf{B}_{L}\left(\mathbf{k}_{i}, 1, \mathbf{x}\right)=\left\{\begin{array}{cc}
\frac{\mathbf{k}_{i} \times \mathbf{e}}{\omega} e^{i \mathbf{k}_{i} \bullet \mathbf{x}}+a_{r} \frac{\mathbf{k}_{r} \times \mathbf{e}}{\omega} e^{i \mathbf{k}_{r} \bullet \mathbf{x}} & , z<0 \\
a_{t} \frac{\mathbf{k}_{t} \times \mathbf{e}}{\omega} e^{i \mathbf{k}_{t} \bullet \mathbf{x}} & , z \geq 0
\end{array}\right.
$$

Similarly, the TE modes normalized upon unit electric field amplitude incoming from the right are labeled by $R$, the incoming wave vector $\mathbf{k}_{i}$ with $K_{i} \leq 0$, and the polarization index 1 , and are given by

$$
\mathbf{E}_{R}\left(\mathbf{k}_{i}, 1, \mathbf{x}\right)=\left\{\begin{array}{cl}
\mathbf{e} a_{t} e^{i \mathbf{k}_{t} \bullet \mathbf{x}} & , z<0 \\
\mathbf{e}\left[e^{i \mathbf{k}_{i} \bullet \mathbf{x}}+a_{r} e^{i \mathbf{k}_{r} \bullet \mathbf{x}}\right] & , z \geq 0
\end{array},\right.
$$

where $\mathbf{e}$ has the same direction as $\mathbf{k}_{i} \times \mathbf{e}_{3}$. The reflexion / transmission coefficients $a_{r}, a_{t}$ have the same form as in (2), but the relation between frequency $\omega$ and wave vectors is now

$$
\mathbf{k}_{\|}^{2}+K_{i}^{2}=\mathbf{k}_{\|}^{2}+K_{r}^{2}=\mu_{0} \epsilon_{0} \omega^{2} \quad, \quad \mathbf{k}_{\|}^{2}+K_{t}^{2}=\mu_{0} \epsilon \omega^{2} .
$$

The associated TE magnetic field is

$$
\mathbf{B}_{R}\left(\mathbf{k}_{i}, 1, \mathbf{x}\right)=\left\{\begin{array}{cc}
a_{t} \frac{\mathbf{k}_{t} \times \mathbf{e}}{\omega} e^{i \mathbf{k}_{t} \bullet \mathbf{x}} & , z<0 \\
\frac{\mathbf{k}_{i} \times \mathbf{e}}{\omega} e^{\mathbf{k}_{i} \bullet \mathbf{x}}+a_{r} \frac{\mathbf{k}_{r} \times \mathbf{e}}{\omega} e^{i \mathbf{k}_{r} \bullet \mathbf{x}} & , z \geq 0
\end{array} .\right.
$$

Now we turn to the TM modes: The magnetic field of the TM modes incoming from the left is

$$
\mathbf{B}_{L}\left(\mathbf{k}_{i}, 2, \mathbf{x}\right)=\left\{\begin{array}{cl}
\mathbf{e}\left[e^{i \mathbf{k}_{i} \bullet \mathbf{x}}+b_{r} e^{i \mathbf{k}_{r} \bullet \mathbf{x}}\right] & , z<0 \\
\mathbf{e} b_{t} e^{i \mathbf{k}_{t} \bullet \mathbf{x}} & , z \geq 0
\end{array}\right.
$$

where $\mathbf{e}$ is a real unit magnetic polarization vector chosen in the direction of $\mathbf{k}_{i} \times \mathbf{e}_{3}$. The associated electric field follows from Maxwell's equations, and is given by

$$
\mathbf{E}_{L}\left(\mathbf{k}_{i}, 2, \mathbf{x}\right)=\left\{\begin{array}{cc}
-\frac{\mathbf{k}_{i} \times \mathbf{e}}{\omega \mu_{0} \epsilon} e^{i \mathbf{k}_{i} \bullet \mathbf{x}}-\frac{\mathbf{k}_{r} \times \mathbf{e}}{\omega \mu_{0} \epsilon} e^{i \mathbf{k}_{r} \bullet \mathbf{x}} & , z<0 \\
-\frac{\mathbf{k}_{t} \times \mathbf{e}}{\omega \mu_{0} \epsilon_{0}} e^{i \mathbf{k}_{t} \bullet \mathbf{x}} & , z \geq 0
\end{array} .\right.
$$

The relation between frequency and wave vectors is given in eq (3). For TM waves incoming from the right we have

$$
\mathbf{B}_{R}\left(\mathbf{k}_{i}, 2, \mathbf{x}\right)=\left\{\begin{array}{cl}
\mathbf{e} b_{t} e^{i \mathbf{k}_{t} \bullet \mathbf{x}} & , z<0 \\
\mathbf{e}\left[e^{i \mathbf{k}_{i} \bullet \mathbf{x}}+b_{r} e^{i \mathbf{k}_{r} \bullet \mathbf{x}}\right] & , z \geq 0
\end{array}\right.
$$

with associated electric field

$$
\mathbf{E}_{R}\left(\mathbf{k}_{i}, 2, \mathbf{x}\right)=\left\{\begin{array}{cc}
-\frac{\mathbf{k}_{t} \times \mathbf{e}}{\omega \mu_{0} \epsilon} e^{i \mathbf{k}_{t} \bullet \mathbf{x}} & , z<0 \\
-\frac{\mathbf{k}_{i} \times \mathbf{e}}{\omega \mu_{0} \epsilon_{0}} e^{i \mathbf{k}_{i} \bullet \mathbf{x}}-\frac{\mathbf{k}_{r} \times \mathbf{e}}{\omega \mu_{0} \epsilon_{0}} e^{i \mathbf{k}_{r} \bullet \mathbf{x}}, & z \geq 0
\end{array} .\right.
$$

The relation between wave vectors and frequency is given in eq (6).

The reflexion / transmission coefficients $b_{r}$ and $b_{t}$ now take a slightly more complicated form in the TM case. In order to avoid the need to explicitly distinguish between left- and right-propagating modes, we introduce the following notation conventions: Let $\mathbb{H}_{i}$ be the half-space in which the incoming component of the triple mode under consideration propagates, and let $\varepsilon_{i}$ and $n_{i}$ be the permittivity and refractive index of this half-space; similarly, denote $\mathbb{H}_{t}$ for the half-space which contains the transmitted component, with associated permittivity $\varepsilon_{t}$ and refractive index $n_{t}$. 
Thus, for waves incoming from the left we have $\mathbb{H}_{i}=\{z<0\}$ and $\mathbb{H}_{t}=\{z \geq 0\}$, as well as $\epsilon_{i}=\epsilon, \epsilon_{t}=\epsilon_{0}$, and $n_{i}=n_{0}, n_{t}=1$; for waves incoming from the right we must interchange $i$ and $t$ in these formulas. With this convention, the reflexion / transmission coefficients for the TM waves can be written in a uniform way as

$$
b_{r}=\frac{K_{i} / \epsilon_{i}-K_{t} / \epsilon_{t}}{K_{i} / \epsilon_{i}+K_{t} / \epsilon_{t}} \quad, \quad b_{t}=2 \frac{K_{i} / \epsilon_{i}}{K_{i} / \epsilon_{i}+K_{t} / \epsilon_{t}}
$$

This result suggests that, in addition to the normal components $K=\mathbf{k} \bullet \mathbf{n}$ of the wave vectors, we also introduce "reduced normal components"

$$
X_{i} \equiv \frac{K_{i}}{n_{i}^{2}}=\frac{\varepsilon_{0}}{\varepsilon_{i}} K_{i} \quad, \quad X_{t} \equiv \frac{K_{t}}{n_{t}^{2}}=\frac{\epsilon_{0}}{\epsilon_{t}} K_{t}
$$

in which case the coefficients (12) can be written as

$$
b_{r}=\frac{X_{i}-X_{r}}{X_{i}+X_{r}} \quad, \quad b_{t}=\frac{2 X_{i}}{X_{i}+X_{r}}
$$

This notational convention will greatly simplify the computational work in the proofs of orthogonality of the various modes.

\section{STRATEGY FOR PROVING ORTHOGONALITY RELATIONS}

We will make use of the following formulas: Let $\rho$ be a complex number. Then

$$
\int_{-\infty}^{0} d x e^{i \rho x}=\frac{-i}{\rho} \quad, \quad \text { for } \operatorname{Im} \rho<0 \quad ; \quad \int_{0}^{\infty} d x e^{i \rho x}=\frac{i}{\rho} \quad, \quad \text { for } \operatorname{Im} \rho>0
$$

As a special case we have

$$
\lim _{\epsilon \rightarrow 0+0} \int_{-\infty}^{0} d x e^{i(k-i \epsilon) x}=\mathcal{P} \frac{-i}{k}+\pi \delta(k) \quad ; \quad \lim _{\epsilon \rightarrow 0+0} \int_{0}^{\infty} d x e^{i(k+i \epsilon) x}=\mathcal{P} \frac{i}{k}+\pi \delta(k)
$$

As the computational work required to prove orthogonality of triple modes is considerable, we seek a path of minimal effort. It turns out that a good strategy goes as follows:

We first prove orthogonality of electric fields associated with TE modes, both for co- and counterpropagating modes; this is done in section [IV. Next we prove orthogonality of magnetic fields associated with TM modes, also for co- and counterpropagating modes; this will be done in section $\mathrm{V}$. Finally, we show that electric as well as magnetic fields associated with one TE and one TM mode always have a vanishing orthogonality integral. This will be done in section $\mathrm{VI}$.

After that, in section VII we will prove the equations

$$
\int d^{3} x\left\{n^{2}(\mathbf{x}) \mathbf{E}^{*}(\mathbf{x}) \bullet \mathbf{E}^{\prime}(\mathbf{x})-c^{2} \mathbf{B}^{*}(\mathbf{x}) \bullet \mathbf{B}^{\prime}(\mathbf{x})\right\}=0
$$

and

$$
\int d^{3} x\left\{n^{2}(x) \mathbf{E}(x) \bullet \mathbf{E}^{\prime}(x)+c^{2} \mathbf{B}(x) \bullet \mathbf{B}^{\prime}(x)\right\}=0,
$$

for all possible TE and TM modes. With the help of $(\sqrt{17})$ we can then immediately find the remaining orthogonality relations: Applying (17) to the results from section IV proves orthogonality of magnetic fields associated with TE modes; while (17) applied to the results of section $\mathrm{V}$ yields orthogonality of electric fields associated with TM modes. Together with (18) we then have completed our task of proving all possible orthogonality relations.

One might wonder why we choose a strategy as contrived as the one above. The reason is simply that this approach minimizes the computational work to be done to prove all the relations discussed above; any other strategy would yield the same results, but would require more effort. 


\section{TE MODES - ORTHOGONALITY OF ELECTRIC FIELDS}

\section{A. Copropagating TE electric fields}

Consider the electric fields of two TE modes

$$
\mathbf{E}\left(\mathbf{k}_{i}, 1, \mathbf{x}\right)=\left\{\begin{array}{cl}
\mathbf{e}\left[e^{i \mathbf{k}_{i} \bullet \mathbf{x}}+a_{r} e^{i \mathbf{k}_{r} \bullet \mathbf{x}}\right] & , \\
\mathbf{e} a_{t} e^{i \mathbf{k}_{t} \bullet \mathbf{x}} & , \mathbf{x} \in \mathbb{H}_{i} \\
& \mathbf{x} \in \mathbb{H}_{t}
\end{array},\right.
$$

and

$$
\mathbf{E}^{\prime}\left(\mathbf{k}_{i}^{\prime}, 1, \mathbf{x}\right)=\left\{\begin{array}{cl}
\mathbf{e}^{\prime}\left[e^{i \mathbf{k}_{i}^{\prime} \bullet \mathbf{x}}+a_{r}^{\prime} e^{i \mathbf{k}_{r}^{\prime} \bullet \mathbf{x}}\right] & , \quad \mathbf{x} \in \mathbb{H}_{i} \\
\mathbf{e}^{\prime} a_{t}^{\prime} e^{i \mathbf{k}_{t}^{\prime} \bullet \mathbf{x}} & , \mathbf{x} \in \mathbb{H}_{t}
\end{array}\right.
$$

where both modes are copropagating, i.e.

$$
\operatorname{sgn}\left(K_{i}\right)=\operatorname{sgn}\left(K_{i}^{\prime}\right)
$$

Then

$$
\int d^{3} x n^{2}(\mathbf{x}) \mathbf{E}\left(\mathbf{k}_{i}, 1, \mathbf{x}\right)^{*} \bullet \mathbf{E}^{\prime}\left(\mathbf{k}_{i}^{\prime}, 1, \mathbf{x}\right)=(2 \pi)^{3} n_{i}^{2} \delta\left(\mathbf{k}_{i}-\mathbf{k}_{i}^{\prime}\right)
$$

and the result holds for both combinations $\mathbf{E}_{L}, \mathbf{E}_{L}^{\prime}$ and $\mathbf{E}_{R}, \mathbf{E}_{R}^{\prime}$ of subscripts. We see that the square of the refractive index serves as a weight function in the integrand. On the right hand side (RHS), $n_{i}$ is the refractive index in the common half-space $\mathbb{H}_{i}$ of the two incident components of the triple modes.

Proof:

We perform the proof for two modes incident from the left. The case of incident waves coming from the right is very similar. If eqs $(19,20)$ are inserted into eq (22), the integration over $x_{1}$ and $x_{2}$ can be performed, which results in a delta function

$$
(2 \pi)^{2} \delta\left(k_{1}^{\prime}-k_{1}\right) \delta\left(k_{2}^{\prime}-k_{2}\right) \equiv(2 \pi)^{2} \delta^{(2)}\left(k_{\|}^{\prime}-k_{\|}\right)
$$

over the parallel components of the wave vectors. Thus we can replace $\mathbf{k}_{\|}^{\prime}$ by $\mathbf{k}_{\|}$in all expressions in the sequel. We write this result as

$$
\int d^{3} x n^{2}(\mathbf{x}) \mathbf{E}(\mathbf{x})^{*} \bullet \mathbf{E}^{\prime}(\mathbf{x})=\left(\mathbf{e} \bullet \mathbf{e}^{\prime}\right)(2 \pi)^{2} \delta^{(2)}\left(\mathbf{k}_{\|}^{\prime}-\mathbf{k}_{\|}\right) \times I
$$

where

$$
\begin{aligned}
I=\int_{-\infty}^{0} d z n_{i}^{2}\left[e^{i z\left(K_{i}^{\prime}-K_{i}\right)}\right. & \left.+a_{r}^{*} e^{i z\left(K_{i}^{\prime}+K_{i}\right)}+a_{r}^{\prime} e^{i z\left(-K_{i}^{\prime}-K_{i}\right)}+a_{r}^{*} a_{r}^{\prime} e^{i z\left(-K_{i}^{\prime}+K_{i}\right)}\right]+ \\
& +\int_{0}^{\infty} d z n_{t}^{2} a_{t}^{*} a_{t}^{\prime} e^{i z\left(K_{t}^{\prime}-K_{t}^{*}\right)}
\end{aligned}
$$

and we have omitted reference to the wave vectors $\mathbf{k}_{i}$ and the polarizations 1,2 in $\mathbf{E}(\mathbf{x})$. Now we apply theorems (15 16) to equation (25). Since $K_{i}, K_{i}^{\prime}$ are always real, the first integral can be treated using theorem (16), resulting in two contributions from the principal part and the delta function. The second integral is more involved: If both $K_{t}$ and $K_{t}^{\prime}$ are real, then theorem (16) gives

$$
\int_{0}^{\infty} d z e^{i z\left(K_{t}^{\prime}-K_{t}^{*}\right)}=\mathcal{P} \frac{i}{K_{t}^{\prime}-K_{t}}+\pi \delta\left(K_{t}^{\prime}-K_{t}\right)
$$

There are three further cases: $K_{t}^{\prime}$ real, $K_{t}=i \kappa_{t} ; K_{t}^{\prime}=i \kappa_{t}^{\prime}, K_{t}$ real; $K_{t}=i \kappa_{t}, K_{t}^{\prime}=i \kappa_{t}^{\prime}$. Since the incident wave comes from the left, the imaginary parts $\kappa_{t}, \kappa_{t}^{\prime}$ must be positive in each case, and hence the condition that $I m\left(K_{t}^{\prime}-K_{t}^{*}\right)>0$ is always fulfilled, so that we can apply theorem (15):

$$
\int_{0}^{\infty} d z e^{i z\left(K_{t}^{\prime}-K_{t}^{*}\right)}=\mathcal{P} \frac{i}{K_{t}^{\prime}-K_{t}^{*}}
$$


In order to treat all four cases simultaneously, we note that the complex delta function $\delta\left(K_{t}^{\prime}-K_{t}^{*}\right)$ is non-zero only in the first case discussed above, i.e. when both $K_{t}^{\prime}$ and $K_{t}$ are real. Hence, results (26) and (27) can be summarized into one formula:

$$
\int_{0}^{\infty} d z e^{i z\left(K_{t}^{\prime}-K_{t}^{*}\right)}=\mathcal{P} \frac{i}{K_{t}^{\prime}-K_{t}^{*}}+\pi \delta\left(K_{t}^{\prime}-K_{t}^{*}\right),
$$

where it is understood that the $\delta$-function contributes only for real argument, and vanishes otherwise. We can now compute expression (25):

$$
\begin{gathered}
I=n_{i}^{2}\left\{\mathcal{P} \frac{-i}{K_{i}^{\prime}-K_{i}}+a_{r}^{*} \mathcal{P} \frac{-i}{K_{i}^{\prime}+K_{i}}+a_{r}^{\prime} \mathcal{P} \frac{i}{K_{i}^{\prime}+K_{i}}+a_{r}^{\prime} a_{r}^{*} \mathcal{P} \frac{i}{K_{i}^{\prime}-K_{i}}\right\}+ \\
+n_{t}^{2} a_{t}^{\prime} a_{t}^{*} \mathcal{P} \frac{i}{K_{t}^{\prime}-K_{t}^{*}}+ \\
+n_{i}^{2} \pi\left\{\delta\left(K_{i}^{\prime}-K_{i}\right)+a_{r}^{*} \delta\left(K_{i}^{\prime}+K_{i}\right)+a_{r}^{\prime} \delta\left(K_{i}^{\prime}+K_{i}\right)+a_{r}^{\prime} a_{r}^{*} \delta\left(K_{i}^{\prime}-K_{i}\right)\right\}+ \\
+\quad n_{t}^{2} \pi a_{t}^{\prime} a_{t}^{*} \delta\left(K_{t}^{\prime}-K_{t}^{*}\right)
\end{gathered}
$$

where we already have split the principal parts from the delta functions. We now must insert (2) for $a_{r}$. A long computation then shows that all principal parts cancel each other: To see this we use

$$
\left(K_{t}^{\prime}-K_{t}^{*}\right)\left(K_{t}^{\prime}+K_{t}^{*}\right)=\left(\omega^{\prime 2}-\omega^{2}\right) \mu_{0} \epsilon_{t}
$$

to derive

$$
\frac{n_{i}^{2}}{\left(K_{i}^{\prime}-K_{i}\right)\left(K_{i}^{\prime}+K_{i}\right)}=\frac{n_{t}^{2}}{\left(K_{t}^{\prime}-K_{t}^{*}\right)\left(K_{t}^{\prime}+K_{t}^{*}\right)}
$$

If the last equation is used in 29), all contributions from principal parts cancel out. Since both $K_{i}^{\prime}$ and $K_{i}$ are positive in the present case, the $\delta\left(K_{i}^{\prime}+K_{i}\right)$-functions in 29$)$ vanish, and we obtain

$$
I=n_{i}^{2} \pi\left\{1+a_{r}^{\prime} a_{r}^{*}\right\} \delta\left(K_{i}^{\prime}-K_{i}\right)+n_{t}^{2} \pi a_{t}^{\prime} a_{t}^{*} \delta\left(K_{t}^{\prime}-K_{t}^{*}\right) .
$$

In this expression, vanishing of the second delta function implies the vanishing of the first. Thus, the only occasion at which $I$ is not zero occurs when both $K_{t}^{\prime}$ and $K_{t}$ are real. Then we can express $\delta\left(K_{t}^{\prime}-K_{t}\right)$ in terms of $\delta\left(K_{i}^{\prime}-K_{i}\right)$,

$$
\delta\left(K_{t}^{\prime}-K_{t}\right)=\frac{n_{i}^{2}}{n_{t}^{2}} \frac{\left|K_{t}\right|}{\left|K_{i}\right|} \delta\left(K_{i}^{\prime}-K_{i}\right)
$$

where we have used the fact that $K_{t}^{\prime}, K_{t}$ as well as $K_{i}, K_{i}^{\prime}$ have the same sign. (32) now becomes

$$
I=\pi n_{i}^{2}\left\{1+a_{r}^{\prime} a_{r}^{*}+a_{t}^{\prime} a_{t}^{*} \frac{\left|K_{t}\right|}{\left|K_{i}\right|}\right\} \delta\left(K_{i}^{\prime}-K_{i}\right)
$$

Since this expression is non-vanishing only for $K_{i}^{\prime}=K_{i}$, we can omit the prime's in the curly brackets. Furthermore $K_{t}, K_{i}$ have the same sign, hence the curly brackets yield

$$
1+a_{r} a_{r}^{*}+a_{t} a_{t}^{*} \frac{K_{t}}{K_{i}}=2
$$

Finally, therefore,

$$
I=2 \pi n_{i}^{2} \delta\left(K_{i}^{\prime}-K_{i}\right) .
$$

If (36) is combined with (24) we arrive at the result (22).

The same result holds for two copropagating modes incident from the right: Again, the square of the index of refraction $n^{2}(\mathbf{x})$ emerges as weight in the integral, while now on the RHS $n_{i}^{2}$ is equal to 1 .

\section{B. Counterpropagating TE electric fields}

In a similar way we prove the orthogonality of two counter-propagating TE modes. It is sufficient to assume that the field $\mathbf{E}$ is incoming from the left, while $\mathbf{E}^{\prime}$ is incident from the right: 
Consider the electric fields of two counter-propagating TE modes

$$
\mathbf{E}_{L}\left(\mathbf{k}_{i}, 1, \mathbf{x}\right)=\left\{\begin{array}{cc}
\mathbf{e}\left[e^{i \mathbf{k}_{i} \bullet \mathbf{x}}+a_{r} e^{i \mathbf{k}_{r} \bullet \mathbf{x}}\right] & , z<0 \\
\mathbf{e} a_{t} e^{i \mathbf{k}_{t} \bullet \mathbf{x}} & , z \geq 0
\end{array}\right.
$$

and

$$
\mathbf{E}_{R}^{\prime}\left(\mathbf{k}_{i}^{\prime}, 1, \mathbf{x}\right)=\left\{\begin{array}{cc}
\mathbf{e}^{\prime} a_{t}^{\prime} e^{i \mathbf{k}_{t}^{\prime} \bullet \mathbf{x}} & , z<0 \\
\mathbf{e}^{\prime}\left[e^{i \mathbf{k}_{i}^{\prime} \bullet \mathbf{x}}+a_{r}^{\prime} e^{i \mathbf{k}_{r}^{\prime} \bullet \mathbf{x}}\right] & , z \geq 0
\end{array}\right.
$$

where $K_{i}>0$ and $K_{i}^{\prime} \leq 0$. Then

$$
\int d^{3} x n^{2}(\mathbf{x}) \mathbf{E}_{L}\left(\mathbf{k}_{i}, 1, \mathbf{x}\right)^{*} \bullet \mathbf{E}_{R}^{\prime}\left(\mathbf{k}_{i}^{\prime}, 1, \mathbf{x}\right)=0
$$

Proof:

Following the same course as in the previous section we find that

$$
\int d^{3} x n^{2}(\mathbf{x}) \mathbf{E}(\mathbf{x})^{*} \bullet \mathbf{E}^{\prime}(\mathbf{x})=(2 \pi)^{2}\left(\mathbf{e} \bullet \mathbf{e}^{\prime}\right) \delta^{(2)}\left(\mathbf{k}_{\|}-\mathbf{k}_{\|}^{\prime}\right) \times I,
$$

where the quantity $I$ now takes the form

$$
\begin{aligned}
I & =n_{0}^{2} \int_{-\infty}^{0} d z\left[a_{t}^{\prime} e^{i z\left(K_{t}^{\prime}-K_{i}\right)}+a_{t}^{\prime} a_{r}^{*} e^{i z\left(K_{t}^{\prime}+K_{i}\right)}\right]+ \\
& +\int_{0}^{\infty} d z\left[a_{t}^{*} e^{i z\left(K_{i}^{\prime}-K_{t}^{*}\right)}+a_{t}^{*} a_{r}^{\prime} e^{i z\left(-K_{i}^{\prime}-K_{t}^{*}\right)}\right]
\end{aligned}
$$

Since the result is non-vanishing only for $\mathbf{k}_{\|}^{\prime}=\mathbf{k}_{\|}$, we have $\mathbf{e} \bullet \mathbf{e}^{\prime}=1$. Integration over $z$ and splitting into principal part and delta functions using eqs (15, 16) gives for the principal-part-contribution to (41)

$$
\begin{gathered}
I_{\text {princ }}=\frac{4 i K_{i}}{\left(K_{i}^{\prime}+K_{t}^{\prime}\right)\left(K_{i}+K_{t}^{*}\right)} \frac{1}{K_{t}^{\prime 2}-K_{i}^{2}} \times \\
\times\left\{-n_{1}^{2} \frac{K_{i}^{\prime}\left(K_{t}^{\prime}+K_{t}^{*}\right)}{K_{t}^{\prime 2}-K_{i}^{2}}+n_{2}^{2} \frac{K_{i}^{\prime}\left(K_{t}^{\prime}+K_{t}^{*}\right)}{K_{i}^{\prime 2}-K_{t}^{* 2}}\right\},
\end{gathered}
$$

while the delta contributions to (41) all vanish, since $K_{i}$ and $K_{i}^{\prime}$ have now opposing sign. Thus, eq (42) can be rewritten as

$$
I_{\text {princ }}=\frac{4 i K_{i} K_{i}^{\prime}\left(K_{t}^{\prime}+K_{t}^{*}\right)}{\left(K_{i}^{\prime}+K_{t}^{\prime}\right)\left(K_{i}+K_{t}^{*}\right)}\left\{-\frac{n_{1}^{2}}{K_{t}^{\prime 2}-K_{i}^{2}}+\frac{n_{2}^{2}}{K_{i}^{\prime 2}-K_{t}^{* 2}}\right\} .
$$

Using the fact that

$$
K_{i}^{\prime 2}-K_{t}^{* 2}=\frac{\varepsilon_{2}}{\varepsilon_{1}}\left(K_{t}^{\prime 2}-K_{i}^{2}\right)
$$

and

$$
n_{2}^{2}=\frac{\varepsilon_{2}}{\varepsilon_{1}} n_{1}^{2}
$$

the curly brackets are seen to vanish, which proves the result.

\section{TM MODES - ORTHOGONALITY OF MAGNETIC FIELDS}

Here we prove analogous relations for TM modes. Since we start with magnetic rather than electric fields, we normalize the incoming magnetic field $\mathbf{B}_{i}$ in such a way that the magnitude of the associated electric field $\mathbf{E}_{i}$ is 1 ,

$$
\left|\mathbf{B}_{i}\right|=\frac{\left|\mathbf{k}_{i}\right|}{\omega}\left|\mathbf{E}_{i}\right|=\frac{1}{c}
$$

Hence, the incoming magnetic plane wave takes the form

$$
\mathbf{B}_{i}=\frac{1}{c} \mathbf{e} e^{i \mathbf{k}_{i} \bullet \mathbf{x}} \quad, \quad|\mathbf{e}|=1 \quad, \quad[\mathbf{e}]=[\mathbf{E}]
$$




\section{A. Copropagating TM magnetic fields}

Consider the magnetic fields of two TM modes

$$
\mathbf{B}\left(\mathbf{k}_{i}, 2, \mathbf{x}\right)=\left\{\begin{array}{cl}
\frac{1}{c} \mathbf{e}\left[e^{i \mathbf{k}_{i} \bullet \mathbf{x}}+b_{r} e^{i \mathbf{k}_{r} \bullet \mathbf{x}}\right] & , \quad \mathbf{x} \in \mathbb{H}_{i} \\
\frac{1}{c} \mathbf{e} b_{t} e^{i \mathbf{k}_{t} \bullet \mathbf{x}} & , \mathbf{x} \in \mathbb{H}_{t}
\end{array},\right.
$$

and

$$
\mathbf{B}^{\prime}\left(\mathbf{k}_{i}^{\prime}, 2, \mathbf{x}\right)=\left\{\begin{array}{cl}
\frac{1}{c} \mathbf{e}^{\prime}\left[e^{i \mathbf{k}_{i}^{\prime} \bullet \mathbf{x}}+b_{r}^{\prime} e^{i \mathbf{k}_{r}^{\prime} \bullet \mathbf{x}}\right] & , \quad \mathbf{x} \in \mathbb{H}_{i} \\
\frac{1}{c} \mathbf{e}^{\prime} b_{t}^{\prime} e^{i \mathbf{k}_{t}^{\prime} \bullet \mathbf{x}} & , \mathbf{x} \in \mathbb{H}_{t}
\end{array}\right.
$$

where both modes are copropagating, i.e.

$$
\operatorname{sgn}\left(K_{i}\right)=\operatorname{sgn}\left(K_{i}^{\prime}\right)
$$

Then

$$
\int d^{3} x \mathbf{B}\left(\mathbf{k}_{i}, 2, \mathbf{x}\right)^{*} \bullet \mathbf{B}^{\prime}\left(\mathbf{k}_{i}^{\prime}, 2, \mathbf{x}\right)=\frac{\mathbf{e}^{2}}{c^{2}}(2 \pi)^{3} \delta\left(\mathbf{k}_{i}-\mathbf{k}_{i}^{\prime}\right),
$$

and the result holds for both combinations $\mathbf{B}_{L}, \mathbf{B}_{L}$ and $\mathbf{B}_{R}, \mathbf{B}_{R}$ of subscripts.

Remark : It is important to note that, in contrast to the electric case (22), in the magnetic case no refractive index appears in the integral (and neither on the RHS of the above equation). The same is true for counterpropagating modes.

Proof:

We proceed similar to the proofs given above. In a first step, we perform the integration over the coordinates $\mathbf{x}_{\|}=\left(x_{1}, x_{2}\right)$ in 49$)$, giving the intermediate result

$$
\int d^{3} x \mathbf{B}(\mathbf{x})^{*} \bullet \mathbf{B}^{\prime}(\mathbf{x})=\left(\frac{2 \pi}{c}\right)^{2}\left(\mathbf{e} \bullet \mathbf{e}^{\prime}\right) \delta^{(2)}\left(\mathbf{k}_{\|}-\mathbf{k}_{\|}^{\prime}\right) \times I
$$

Due to the delta factor we have $\mathbf{e} \bullet \mathbf{e}^{\prime}=1$. As before, the expression $I$ can be written as the sum of two parts $I_{\text {delta }}+I_{\text {princ }}$, involving the delta functions and the principal values, respectively. The principal part contribution vanishes as in the previous computations, $I_{\text {princ }}=0$. The delta contribution is

$$
I_{\text {delta }}=\pi\left\{b_{t}^{*} b_{t}^{\prime} \delta\left(K_{t}^{\prime}-K_{t}\right)+\delta\left(K_{i}^{\prime}-K_{i}\right)+b_{r}^{\prime} b_{r}^{*} \delta\left(K_{i}^{\prime}-K_{i}\right)\right\}
$$

where the first term involving $\delta\left(K_{t}^{\prime}-K_{t}\right)$ is present only if both $K_{t}$ and $K_{t}^{\prime}$ are real. If either of them is imaginary then according to the previous discussion the first term vanishes.

Next we use the fact that the modes are copropagating,

$$
\operatorname{sgn}\left(K_{i}\right)=\operatorname{sgn}\left(K_{i}^{\prime}\right)=\operatorname{sgn}\left(K_{t}\right)=\operatorname{sgn}\left(K_{t}^{\prime}\right)
$$

to obtain

$$
\delta\left(K_{t}^{\prime}-K_{t}\right)=\frac{X_{t}}{X_{i}} \delta\left(K_{i}^{\prime}-K_{i}\right)
$$

provided that both $K_{t}$ and $K_{t}^{\prime}$ are real. The quantities $X$ are defined in eq (13).

Now, three cases must be distinguished: (1), both $K_{t}$ and $K_{t}^{\prime}$ are real; (2), both $K_{t}$ and $K_{t}^{\prime}$ are imaginary; (3), one of them is real and the other one is imaginary. We first deal with case (3): We have the equation

$$
K_{t}^{\prime 2}-K_{t}^{2}=\frac{\epsilon_{t}}{\epsilon_{i}}\left(K_{i}^{\prime 2}-K_{i}^{2}\right)
$$

In case (3), the LHS is always nonvanishing, and so is the RHS. But this means that certainly

$$
K_{i} \neq K_{i}^{\prime}
$$

hence all delta functions involving $K_{i}$ and $K_{i}^{\prime}$ vanish in (51). Furthermore, the first delta function involving $K_{t}$ and $K_{t}^{\prime}$ vanishes naturally in this case, as was explained in section IV. It follows that the orthogonality integral must vanish in this case, and so eq (49) is confirmed. 
Now we turn to case (1), where $K_{t}$ and $K_{t}^{\prime}$ are real. Here we find on using (53) that

$$
I_{\text {delta }}=\pi \delta\left(K_{i}^{\prime}-K_{i}\right)\left\{b_{t}^{2} \frac{X_{t}}{X_{i}}+1+b_{r}^{2}\right\} .
$$

Direct computation now shows that the curly brackets yield 2, and therefore

$$
I_{\text {delta }}=2 \pi \delta\left(K_{i}^{\prime}-K_{i}\right)
$$

(57) together with (50) now confirms (49).

Finally, we consider the case where both $K_{t}$ and $K_{t}^{\prime}$ are imaginary, and hence are associated with evanescent modes. Now we have $K_{t}^{*}=-K_{t}$, and (51) gives

$$
I_{\text {delta }}=\pi \delta\left(K_{i}^{\prime}-K_{i}\right)\left\{1+\left|b_{r}\right|^{2}\right\} .
$$

But

$$
\left|b_{r}\right|^{2}=\frac{\left|X_{i}-X_{t}\right|^{2}}{\left|X_{i}+X_{t}\right|^{2}}=1
$$

due to the fact that $X_{i}$ is real, and $X_{t}$ is purely imaginary, $X_{t}^{*}=-X_{t}$. Thus, again we find (57) for $I_{\text {delta }}$, which confirms (49).

\section{B. Counterpropagating TM magnetic fields}

This case proceeds exactly analogous to the previous results, so we quote the result without explicit proof: The magnetic fields of two counterpropagating TM modes,

$$
\operatorname{sgn}\left(K_{i}\right)=-\operatorname{sgn}\left(K_{i}^{\prime}\right),
$$

are orthogonal,

$$
\int d^{3} x \mathbf{B}_{L}\left(\mathbf{k}_{i}, 2, \mathbf{x}\right)^{*} \bullet \mathbf{B}_{R}^{\prime}\left(\mathbf{k}_{i}^{\prime}, 2, \mathbf{x}\right)=0
$$

\section{ONE TE- , ONE TM MODE}

Finally, we show that a pair of modes such that one member is TE and the other one is TM, is always orthogonal. This holds for both electric and magnetic fields:

$$
\begin{aligned}
\int d^{3} x n^{2}(x) \mathbf{E}\left(\mathbf{k}_{i}, 1, \mathbf{x}\right)^{*} \bullet \mathbf{E}^{\prime}\left(\mathbf{k}_{i}^{\prime}, 2, \mathbf{x}\right) & =0 \\
\int d^{3} x \mathbf{B}\left(\mathbf{k}_{i}, 1, \mathbf{x}\right)^{*} \bullet \mathbf{B}^{\prime}\left(\mathbf{k}_{i}^{\prime}, 2, \mathbf{x}\right) & =0 .
\end{aligned}
$$

These results hold for all combinations of subscripts $L L, L R, R L$ and $R R$.

Proof:

From the results above, we know that each of the integrals $J$ in eqs $(62,63)$ has the form

$$
J=(2 \pi)^{2} \delta^{(2)}\left(\mathbf{k}_{\|}-\mathbf{k}_{\|}^{\prime}\right) \times I \quad,
$$

which follows from performing the integration in (62) over the two coordinates $\mathbf{x}_{\|}$. Because of the delta factor, both wave vectors lie in the same plane of incidence. First, consider the electric case in (62), and assume that $\mathbf{E}$ is TE, and $\mathbf{E}^{\prime}$ is TM. Then $\mathbf{E}$ is perpendicular to the plane of incidence, while $\mathbf{E}^{\prime}$ lies in the plane, hence the LHS of (62) vanishes. - The same argument immediately applies to the magnetic case. 


\section{A THEOREM LINKING ELECTRIC AND MAGNETIC ORTHOGONALITY}

Now we turn to prove the following equations,

$$
\begin{gathered}
\int d^{3} x\left\{n^{2}(x) \mathbf{E}^{*}(x) \bullet \mathbf{E}^{\prime}(x)-c^{2} \mathbf{B}^{*}(x) \bullet \mathbf{B}^{\prime}(x)\right\}=0, \\
\int d^{3} x\left\{n^{2}(x) \mathbf{E}(x) \bullet \mathbf{E}^{\prime}(x)+c^{2} \mathbf{B}(x) \bullet \mathbf{B}^{\prime}(x)\right\}=0,
\end{gathered}
$$

valid for all combinations $L L, L R, R L$ and $R R$ of subscripts as well as for both TE and TM polarizations. These equations will complete our discussion of orthogonality of triple modes.

Remark: Note that the first of these equations involves one complex conjugate of an electric and a magnetic field, respectively, while the second equation contains no complex conjugate fields.

Proof:

We first prove 6 65).

We start by introducing the explicit time dependence of the modes,

$$
\begin{aligned}
\mathbf{E} \equiv \mathbf{E}(x) e^{-i \omega t} & \quad \mathbf{B} \equiv \mathbf{B}(x) e^{-i \omega t} \\
\mathbf{E}^{\prime} \equiv \mathbf{E}^{\prime}(x) e^{-i \omega^{\prime} t} & , \quad \mathbf{B}^{\prime} \equiv \mathbf{B}^{\prime}(x) e^{-i \omega^{\prime} t}
\end{aligned}
$$

These time-dependent fields must obey Maxwell's equations, from which we derive

$$
\nabla \bullet\left[\mathbf{E}^{*} \times \mathbf{B}^{\prime}\right]=i\left\{\omega^{\prime} \frac{n^{2}}{c^{2}} \mathbf{E}^{*} \bullet \mathbf{E}^{\prime}-\omega \mathbf{B}^{*} \bullet \mathbf{B}^{\prime}\right\} .
$$

This equation contains a time dependence $\exp i\left(\omega-\omega^{\prime}\right) t$ on both sides. Integrating over $t$ and $\omega^{\prime}$ yields

$$
\frac{1}{\omega} \nabla \bullet\left[\mathbf{E}^{*}(x) \times \mathbf{B}^{\prime}(x)\right]=i\left\{\frac{n^{2}}{c^{2}} \mathbf{E}^{*}(x) \bullet \mathbf{E}^{\prime}(x)-\mathbf{B}^{*}(x) \bullet \mathbf{B}^{\prime}(x)\right\},
$$

where $\mathbf{E}(x)$ etc are again triple mode functions. In order to prove (65) we therefore must show that

$$
\int d^{3} x \nabla \bullet\left[\mathbf{E}^{*}(x) \times \mathbf{B}^{\prime}(x)\right]=0
$$

for any two triple modes $\mathbf{E}(x)$ and $\mathbf{B}^{\prime}(x)$. To see this we first note that the spatial dependence of the integrand can be written as

$$
\mathbf{E}^{*}(x) \times \mathbf{B}^{\prime}(x)=\mathbf{C}(z) e^{i \mathbf{x}_{\|} \bullet\left(\mathbf{k}_{\|}^{\prime}-\mathbf{k}_{\|}\right)},
$$

where $\mathbf{C}(z)$ is given by the expression

$$
\mathbf{C}(z)=e^{-i \mathbf{x}_{\|} \bullet\left(\mathbf{k}_{\|}^{\prime}-\mathbf{k}_{\|}\right)}\left[\mathbf{E}^{*}(x) \times \mathbf{B}^{\prime}(x)\right] .
$$

A brief investigation shows that $\mathbf{C}(z)$ indeed depends only on $z$, as suggested by the notation. Insertion into (70) yields

$$
\int d^{3} x \nabla \bullet\left[\mathbf{E}^{*}(x) \times \mathbf{B}^{\prime}(x)\right]=(2 \pi)^{2} \delta^{(2)}\left(\mathbf{k}_{\|}^{\prime}-\mathbf{k}_{\|}\right) \int d z\left[\partial_{z} \mathbf{C}(z)\right] .
$$

It follows that we must show the vanishing of the surface integral

$$
\int d z\left[\partial_{z} \mathbf{C}(z)\right]=0 \quad, \quad \text { for } \quad \mathbf{k}_{\|}^{\prime}=\mathbf{k}_{\|} .
$$

We see immediately from eq (72) that, if $\mathbf{E}$ is TE and $\mathbf{B}^{\prime}$ is TM, then $\mathbf{C}$ and hence the integral (73) vanish as required. To show that the same holds if $\mathbf{E}$ is TM and $\mathbf{B}^{\prime}$ is TE, we take the complex conjugate of eq (69),

$$
\frac{1}{\omega} \nabla \bullet\left[\mathbf{E}^{*}(x) \times \mathbf{B}^{\prime}(x)\right]=\left\{-\frac{1}{\omega^{\prime}} \nabla \bullet\left[\mathbf{E}^{\prime *}(x) \times \mathbf{B}(x)\right]\right\}^{*} .
$$

Since by assumption, $\mathbf{E}^{\prime}$ and $\mathbf{B}$ are parallel, the RHS of (75) vanishes, which proves the above statement.

Thus, we are left to prove the following four cases: 
1. E and $\mathbf{B}^{\prime}$ TE, copropagating;

2. E and $\mathbf{B}^{\prime}$ TE, counter-propagating;

3. E and $\mathbf{B}^{\prime} \mathrm{TM}$, copropagating;

4. $\mathbf{E}$ and $\mathbf{B}^{\prime}$ TM, counter-propagating.

Due to the $\delta^{(2)}\left(\mathbf{k}_{\|}^{\prime}-\mathbf{k}_{\|}\right)$factor in $(73)$, we can assume that both wave vectors lie in the same plane of incidence. This implies, in particular, that in cases (1) and (2), the magnetic field $\mathbf{B}^{\prime}$ lies in the common plane of incidence, while $\mathbf{E}$ is perpendicular to it; and in cases (3) and (4), the electric field $\mathbf{E}$ lies in the plane of incidence, while $\mathbf{B}^{\prime}$ is perpendicular.

Let us now treat case (1): For the sake of simplicity, we assume that both modes propagate from the left to the right, i.e. $K_{i}, K_{i}^{\prime}>0$. We decompose the divergence in eq (73) into parallel and normal derivatives,

$$
\nabla \bullet\left(\mathbf{E}^{*} \times \mathbf{B}^{\prime}\right)=\nabla_{\|} \bullet\left(\mathbf{E}^{*} \times \mathbf{B}^{\prime}\right)_{\|}+\partial_{3}\left(\mathbf{E}^{*} \times \mathbf{B}^{\prime}\right)_{3}
$$

A computation shows that integration of the first term on the RHS of (76) over the spatial coordinates vanishes because of property (75). In the second term, all terms involving delta functions vanish. Evaluating the principal value contributions gives

$$
\begin{gathered}
\int d^{3} x \omega^{\prime} \partial_{3}\left(\mathbf{E}^{*} \times \mathbf{B}^{\prime}\right)_{3}=(2 \pi)^{2} \delta^{(2)}\left(\mathbf{k}_{\|}^{\prime}-\mathbf{k}_{\|}\right) \omega^{\prime} \cdot\left\{K_{i}^{\prime}\left\{1-a_{r}^{\prime}+a_{r}^{*}-a_{r}^{\prime} a_{r}^{*}\right\}-\right. \\
\left.-K_{t}^{\prime} a_{t}^{\prime} a_{t}^{*}\right\}
\end{gathered}
$$

On account of

$$
1-a_{r}^{\prime}+a_{r}^{*}-a_{r}^{\prime} a_{r}^{*}=\frac{K_{t}^{\prime}}{K_{i}^{\prime}} a_{t}^{\prime} a_{t}^{*},
$$

we find that the RHS of (77) must vanish. The statement that integral (73) vanishes is therefore confirmed, for case (1).

Now we briefly discuss the remaining three cases: Case (2) proceeds along the same lines as case (1). To prove cases (3) and (4) we start with

$$
\nabla \bullet\left(\mathbf{E}^{*} \times \mathbf{B}^{\prime}\right)=-\nabla \bullet\left(\mathbf{B}^{\prime} \times \mathbf{E}^{*}\right)
$$

On the RHS, we express $\mathbf{E}$ by the associated magnetic field $\mathbf{B}$, which is TM,

$$
\mathbf{E}_{i}=-\frac{\mathbf{k}_{i} \times \mathbf{B}_{i}}{\omega \mu_{0} \epsilon_{i}} \quad, \quad \mathbf{E}_{r}=-\frac{\mathbf{k}_{r} \times \mathbf{B}_{r}}{\omega \mu_{0} \epsilon_{i}} \quad, \quad \mathbf{E}_{t}=-\frac{\mathbf{k}_{t} \times \mathbf{B}_{t}}{\omega \mu_{0} \epsilon_{t}}
$$

Subsequent evaluation of the integral then yields a factor $(2 \pi)^{2} \delta^{(2)}\left(\mathbf{k}_{\|}^{\prime}-\mathbf{k}_{\|}\right)$times an expression $\frac{1}{\mu_{0}} f\left(X_{i}, X_{t}, X_{i}^{\prime}, X_{t}^{\prime}, b_{r}, b_{t}, b_{r}^{\prime}, b_{t}^{\prime}\right)$, where $f$ as a function of the arguments $X$ and $b$ has the same functional form as the analogous expression in the TE case as a function of respective TE quantities $K$ and $a$,

$$
f\left(X_{i}, X_{t}, X_{i}^{\prime}, X_{t}^{\prime}, b_{r}, b_{t}, b_{r}^{\prime}, b_{t}^{\prime}\right) \leftrightarrow f\left(K_{i}, K_{t}, K_{i}^{\prime}, K_{t}^{\prime}, a_{r}, a_{t}, a_{r}^{\prime}, a_{t}^{\prime}\right)
$$

Now, the functional form of the quantities $b$ in terms of $X$ is the same as the functional form of $a$ in terms of $K$, as can be seen by comparing eqs (2) and (13). It follows that we may transfer all conclusions derived previously for the TE case to the TM case by making the replacements indicated in the last formula (81). In particular, vanishing $f$ on the RHS of (81) implies vanishing $f$ on the LHS. In this way, we have traced back cases $(3,4)$ to cases $(1,2)$, which finishes the proof of 65 ).

- Now we can turn to the proof of eq (66). This proceeds analogous to the previous equation (65), hence we give only an outline: By using the explicit time dependence (67) of the modes we can derive the relation

$$
\frac{1}{\omega} \nabla \bullet\left[\mathbf{E}(x) \times \mathbf{B}^{\prime}(x)\right]=i\left\{\frac{n^{2}}{c^{2}} \mathbf{E}(x) \bullet \mathbf{E}^{\prime}(x)+\mathbf{B}(x) \bullet \mathbf{B}^{\prime}(x)\right\}
$$

between triple mode functions, which is analogous to eq (69). To prove (66) we therefore must show that

$$
\int d^{3} x \nabla \bullet\left[\mathbf{E}(x) \times \mathbf{B}^{\prime}(x)\right]=0,
$$


which is analogous to (70). The left hand side of the last equation can again be cast into a form similar to (73),

$$
\int d^{3} x \nabla \bullet\left[\mathbf{E}^{*}(x) \times \mathbf{B}^{\prime}(x)\right]=(2 \pi)^{2} \delta^{(2)}\left(\mathbf{k}_{\|}^{\prime}+\mathbf{k}_{\|}\right) \int d z\left[\partial_{z} \mathbf{C}(z)\right],
$$

except that the sign of the second wave vector in the argument of the delta function now differs. It follows that we must show the vanishing of the surface integral

$$
\int d z\left[\partial_{z} \mathbf{C}(z)\right]=0 \quad, \quad \text { for } \quad \mathbf{k}_{\|}^{\prime}=-\mathbf{k}_{\|}
$$

which is analogous to (74). The remainder of the proof proceeds along the same lines as the proof for (65). This finishes our proof of eqs $(65,66)$.

- The remaining orthogonality relations now follow from (65): Suppose that $\mathbf{E}$ and $\mathbf{E}^{\prime}$ are electric fields associated with TM modes; then orthogonality follows from orthogonality of the magnetic fields as proven in section $\mathrm{V}$ and (65). On the other hand, suppose that $\mathbf{B}$ and $\mathbf{B}^{\prime}$ are magnetic fields associated with TE modes; then their orthogonality follows from orthogonality of the associated electric fields as proven in section [V] and again eq (65). This finishes our proof of orthogonality relations.

\section{NORMALIZED MODES}

In the developments above, we have normalized the electric field of TE modes upon unit amplitude of the incoming plane wave, and the magnetic field of TM modes upon a value numerically equal to $1 / c$ for the incoming plane wave. This choice was motivated by simplification of the subsequent computational work. Now we finally introduce a normalization which is best suited for the purpose of diagonalization of the free field Hamiltonian, as performed below. The new normalization will affect only TE modes; in this case, eq (22) motivates that we divide these modes by the refractive index $n_{i}$ of the medium in the incoming half-space $\mathbb{H}_{i}$. If the TE mode under consideration is incoming from the right, the associated refractive index is actually 1, so that these modes are unaffected. Thus, the new normalization effectively changes only the TE modes incoming from the left.

The new TE modes are thus defined as

$$
\mathbf{E}_{L}\left(\mathbf{k}_{i}, 1, \mathbf{x}\right) \equiv\left\{\begin{array}{cc}
\frac{1}{n_{0}} \mathbf{e}\left[e^{i \mathbf{k}_{i} \bullet \mathbf{x}}+a_{r} \cdot e^{i \mathbf{k}_{r} \bullet \mathbf{x}}\right] & , z<0 \\
\frac{1}{n_{0}} \mathbf{e} \cdot a_{t} \cdot e^{i \mathbf{k}_{t} \bullet \mathbf{x}} & , z \geq 0
\end{array}\right.
$$

and

$$
\mathbf{E}_{R}\left(\mathbf{k}_{i}, 1, \mathbf{x}\right) \equiv\left\{\begin{array}{cl}
\mathbf{e}\left[e^{i \mathbf{k}_{i} \bullet \mathbf{x}}+a_{r} \cdot e^{i \mathbf{k}_{r} \bullet \mathbf{x}}\right] & , z \geq 0 \\
\mathbf{e} \cdot a_{t} \cdot e^{i \mathbf{k}_{t} \bullet \mathbf{x}} & , z<0
\end{array}\right.
$$

with

$$
a_{r}=\frac{K_{i}-K_{t}}{K_{i}+K_{t}} \quad, \quad a_{t}=\frac{2 K_{i}}{K_{i}+K_{t}}
$$

Furthermore, $\mathbf{B}\left(\mathbf{k}_{i}, 1, \mathbf{x}\right)$ is defined to be the magnetic field associated with (86, 87), where each of the plane wave components of $\mathbf{B}$ is given in terms of the plane wave components of $\mathbf{E}$ according to

$$
\mathbf{B}_{i}=\frac{\mathbf{k}_{i} \times \mathbf{E}_{i}}{\omega} \quad, \quad \mathbf{B}_{r}=\frac{\mathbf{k}_{r} \times \mathbf{E}_{r}}{\omega} \quad, \quad \mathbf{B}_{t}=\frac{\mathbf{k}_{t} \times \mathbf{E}_{t}}{\omega}
$$

Formulas $(88,89)$ are valid for both left- and right-incoming modes. This defines the normalized TE modes.

The normalized TM modes are given by

$$
\mathbf{B}_{L}\left(\mathbf{k}_{i}, 2, \mathbf{x}\right)=\left\{\begin{array}{cc}
\frac{1}{c} \mathbf{e}\left[e^{i \mathbf{k}_{i} \bullet \mathbf{x}}+b_{L r} \cdot e^{i \mathbf{k}_{r} \bullet \mathbf{x}}\right] & , z<0 \\
\frac{1}{c} \mathbf{e} \cdot b_{L t} \cdot e^{i \mathbf{k}_{t} \bullet \mathbf{x}} & , z \geq 0
\end{array}\right.
$$

where the reflection and transmission coefficients follow from eqs (12 14),

$$
b_{L r}=\frac{K_{i}-n_{0}^{2} K_{t}}{K_{i}+n_{0}^{2} K_{t}} \quad, \quad b_{L t}=\frac{2 K_{i}}{K_{i}+n_{0}^{2} K_{t}}
$$


and for modes incoming from the right,

$$
\mathbf{B}_{R}\left(\mathbf{k}_{i}, 2, \mathbf{x}\right)=\left\{\begin{array}{cl}
\frac{1}{c} \mathbf{e}\left[e^{i \mathbf{k}_{i} \bullet \mathbf{x}}+b_{R r} \cdot e^{i \mathbf{k}_{r} \bullet \mathbf{x}}\right] & , z \geq 0 \\
\frac{1}{c} \mathbf{e} \cdot b_{R t} \cdot e^{i \mathbf{k}_{t} \bullet \mathbf{x}} & , z<0
\end{array}\right.
$$

with

$$
b_{R r}=\frac{n_{0}^{2} K_{i}-K_{t}}{n_{0}^{2} K_{i}+K_{t}} \quad, \quad b_{R t}=\frac{2 K_{i}}{K_{i}+\frac{K_{t}}{n_{0}^{2}}}
$$

The plane wave components of the associated TM electric fields are given in terms of plane wave components of the TM magnetic fields: For waves incoming from the left we have

$$
\mathbf{E}_{L i}=-\frac{c^{2}}{n_{0}^{2}} \frac{\mathbf{k}_{i} \times \mathbf{B}_{i}}{\omega} \quad, \quad \mathbf{E}_{L r}=-\frac{c^{2}}{n_{0}^{2}} \frac{\mathbf{k}_{r} \times \mathbf{B}_{r}}{\omega} \quad, \quad \mathbf{E}_{L t}=-c^{2} \frac{\mathbf{k}_{t} \times \mathbf{B}_{t}}{\omega},
$$

while for waves incoming from the right,

$$
\mathbf{E}_{R i}=-c^{2} \frac{\mathbf{k}_{i} \times \mathbf{B}_{i}}{\omega} \quad, \quad \mathbf{E}_{R r}=-c^{2} \frac{\mathbf{k}_{r} \times \mathbf{B}_{r}}{\omega} \quad, \quad \mathbf{E}_{R t}=-\frac{c^{2}}{n_{0}^{2}} \frac{\mathbf{k}_{t} \times \mathbf{B}_{t}}{\omega} .
$$

All orthogonality relations discussed before can now summarized:

$$
\begin{gathered}
\int d^{3} x n^{2}(x) \mathbf{E}^{*}(\mathbf{k}, s, \mathbf{x}) \bullet \mathbf{E}^{\prime}\left(\mathbf{k}^{\prime}, s^{\prime}, \mathbf{x}\right)=(2 \pi)^{3} \delta_{s s^{\prime}} \delta\left(\mathbf{k}^{\prime}-\mathbf{k}\right) \\
\int d^{3} x \mathbf{B}^{*}(\mathbf{k}, s, \mathbf{x}) \bullet \mathbf{B}^{\prime}\left(\mathbf{k}^{\prime}, s^{\prime}, \mathbf{x}\right)=(2 \pi)^{3} \frac{1}{c^{2}} \delta_{s s^{\prime}} \delta\left(\mathbf{k}_{i}-\mathbf{k}_{i}^{\prime}\right)
\end{gathered}
$$

for both combinations $L L$ and $R R$, and

$$
\begin{gathered}
\int d^{3} x n^{2}(x) \mathbf{E}_{L}^{*}(\mathbf{k}, s, \mathbf{x}) \bullet \mathbf{E}_{R}^{\prime}\left(\mathbf{k}^{\prime}, s^{\prime}, \mathbf{x}\right)=0 \\
\int d^{3} x \mathbf{B}_{L}^{*}(\mathbf{k}, s, \mathbf{x}) \bullet \mathbf{B}_{R}^{\prime}\left(\mathbf{k}^{\prime}, s^{\prime}, \mathbf{x}\right)=0
\end{gathered}
$$

Furthermore, eqs 65, 66) continue to hold.

\section{EXPANSION OF ARBITRARY SOURCE-FREE FIELDS IN TERMS OF TRIPLE MODES}

The set of triple modes is complete with respect to the source-free fields, as has been shown in [14. This means that every source-free electric / magnetic field can be uniquely expressed as a linear combination of triple modes. For an arbitrary free real electric field $\mathbf{E}(\mathbf{x}, t)$ we can make the ansatz

$$
\begin{gathered}
\mathbf{E}(\mathbf{x}, t)= \\
=\quad i \int_{K<0} d^{3} k \sum_{s=1}^{2} \mathcal{E}(k)\left[u_{L s}(\mathbf{k}) \mathbf{E}_{L}(\mathbf{k}, s, \mathbf{x}) e^{-i \omega t}-u_{L s}^{*}(\mathbf{k}) \mathbf{E}_{L}^{*}(\mathbf{k}, s, \mathbf{x}) e^{i \omega t}\right] \\
+i \int_{K \geq 0} d^{3} k \sum_{s=1}^{2} \mathcal{E}(k)\left[u_{R s}(\mathbf{k}) \mathbf{E}_{R}(\mathbf{k}, s, \mathbf{x}) e^{-i \omega t}-u_{R s}^{*}(\mathbf{k}) \mathbf{E}_{R}^{*}(\mathbf{k}, s, \mathbf{x}) e^{i \omega t}\right]
\end{gathered}
$$

where

$$
\mathcal{E}(k) \equiv \sqrt{\frac{\hbar c|\mathbf{k}|}{2 \epsilon_{0}(2 \pi)^{3}}} .
$$


The associated magnetic field is determined by Maxwell's equation $\nabla \times \mathbf{E}=-\dot{\mathbf{B}}$, and has the expansion

$$
\begin{gathered}
\mathbf{B}(\mathbf{x}, t)= \\
=i \int_{K<0} d^{3} k \sum_{s=1}^{2} \mathcal{E}(k)\left[u_{L s}(\mathbf{k}) \mathbf{B}_{L}(\mathbf{k}, s, \mathbf{x}) e^{-i \omega t}-u_{L s}^{*}(\mathbf{k}) \mathbf{B}_{L}^{*}(\mathbf{k}, s, \mathbf{x}) e^{i \omega t}\right] \\
+i \int_{K \geq 0} d^{3} k \sum_{s=1}^{2} \mathcal{E}(k)\left[u_{R s}(\mathbf{k}) \mathbf{B}_{R}(\mathbf{k}, s, \mathbf{x}) e^{-i \omega t}-u_{R s}^{*}(\mathbf{k}) \mathbf{B}_{R}^{*}(\mathbf{k}, s, \mathbf{x}) e^{i \omega t}\right]
\end{gathered}
$$

We note that the amplitude factor $\mathcal{E}(k)$ is the same as in formula (100).

\section{EXPANSION OF THE HAMILTONIAN IN TERMS OF TRIPLE MODES}

The Hamiltonian of the source-free radiation field is based on the classical electromagnetic field energy

$$
H=\frac{\epsilon_{0}}{2} \int d^{3} x\left[n^{2}(\mathbf{x}) \mathbf{E}^{2}(\mathbf{x}, t)+c^{2} \mathbf{B}^{2}(\mathbf{x}, t)\right],
$$

with real fields $\mathbf{E}$ and $\mathbf{B}$. If we insert expansions (100, 102) into (103), we obtain the mode expansion of the Hamiltonian

$$
\begin{aligned}
H & =\int_{K<0} d^{3} k \sum_{s=1}^{2} \frac{\hbar c|\mathbf{k}|}{2}\left[u_{L s}(\mathbf{k}) u_{L s}^{*}(\mathbf{k})+u_{L s}^{*}(\mathbf{k}) u_{L s}(\mathbf{k})\right] \\
& +\int_{K \geq 0} d^{3} k \sum_{s=1}^{2} \frac{\hbar c|\mathbf{k}|}{2}\left[u_{R s}(\mathbf{k}) u_{R s}^{*}(\mathbf{k})+u_{R s}^{*}(\mathbf{k}) u_{R s}(\mathbf{k})\right]
\end{aligned}
$$

To arrive at this expression, we had to employ all orthogonality relations $(96,99)$ and (65, 66 ).

In our current discussion, the coefficients $u_{L R, s}$ are $c$-numbers, as we are still in the classical domain. In deriving the mode expansion (104), we have been careful, however, to retain the order in which terms appear, so that (104)

can be immediately transferred into the quantum regime, by interpreting $u_{L s}(\mathbf{k}), u_{R s}^{\dagger}(\mathbf{k})$ etc. as annihilation and creation operators of triple modes.

\section{SUMMARY}

We have worked out the orthogonality relations for the set of Carniglia-Mandel triple modes, which provide a set of normal modes for the source-free electromagnetic field in a background consisting of a dielectric half-space and the vacuum, respectively. The inherent computational complexity in this kind of problem creates a demand for efficient strategies to accomplish this task. In this paper we have provided this strategy, and furthermore, we have presented a comprehensive discussion of the proofs for the various distinct cases in the orthogonality integral in order to serve as a compilation of technical details for related problems in the field of optics with possibly even greater technical complexity.

\section{ACKNOWLEDGEMENT}

Hanno Hammer wishes to acknowledge support from the Weizmann Institute of Sciences.

[1] Purcell, E. M., 1946, Phys. Rev., 69, 681.

[2] Jaynes, E. T., and Cummings, F. W., 1963, IEEE J. Quant. Elec., 51, 89.

[3] Stehle, P., 1970, Phys. Rev. A, 2, 102. 
[4] Barton, G., 1970, Proc. Royal Soc. London A, 320, 251.

[5] Hinds, E. A., 1991, Cavity Quantum Electrodynamics. Advances in Atomic, Molecular and Optical Physics, 28, $237-286$.

[6] Berman, P. R. (ed.), 1994, Cavity Quantum Electrodynamics (San Diego: Academic Press).

[7] Carniglia, C. K., and Mandel, L., 1971, Phys. Rev. D, 3, 280.

[8] Carniglia, C. K., Mandel, L., and Drexhage, K. H., 1972, J. Opt. Soc. Am., 62, 479.

[9] M. Janowicz and W. Żakowicz, 1994, Phys. Rev. A, 50, 4350.

[10] Inoue, T., and Hori, H., 2001, Phys. Rev. A, 63, 063805.

[11] Urbach, H. P., and Rikken, G. L. J. A., 1998, Phys. Rev. A, 57, 3913.

[12] Żakowicz, W., and Bledowski, A., 1995, Phys. Rev. A, 52, 1640.

[13] Glauber, R. J., and Lewenstein, M., 1991, Phys. Rev. A, 43, 467.

[14] Bialynicki-Birula, I., and Brojan, J. B., 1972, Phys. Rev. D, 5, 485.

[15] Jackson, J. D., 1999, Classical Electrodynamics (New York: John Wiley), 3rd ed.

[16] Born, M., and Wolf, E., 1970, Principles of Optics (Oxford: Pergamon), 4th ed. 